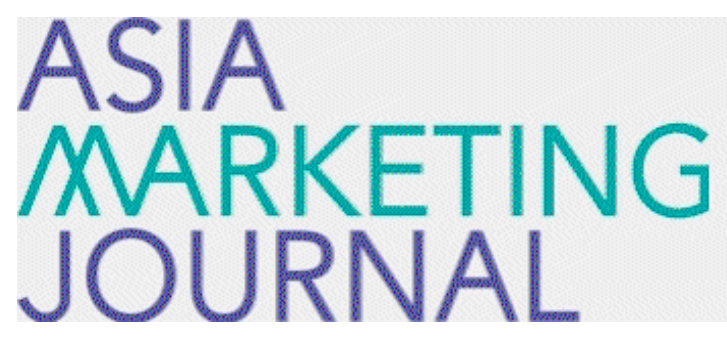

ASIA MARKETING JOURNAL

Volume 22 | Issue 1

Article 2

4-30-2020

\title{
Muscling My Way to My Positive Future: Physical Exertion of Strength and Preference for Risk
}

Eunice Kim Cho

Hee-Kyung Ahn

Follow this and additional works at: https://amj.kma.re.kr/journal

Part of the Marketing Commons

\section{Recommended Citation}

Cho, Eunice Kim and Ahn, Hee-Kyung (2020) "Muscling My Way to My Positive Future: Physical Exertion of Strength and Preference for Risk," Asia Marketing Journal: Vol. 22 : Iss. 1 , Article 2.

Available at: https://doi.org/10.15830/amj.2020.22.1.27

This Article is brought to you for free and open access by Asia Marketing Journal. It has been accepted for inclusion in Asia Marketing Journal by an authorized editor of Asia Marketing Journal. 


\title{
Muscling My Way to My Positive Future: Physical Exertion of Strength and Preference for Risk
}

\author{
Eunice Kim Cho* \\ Hee-Kyung Ahn**
}

\begin{abstract}
Building on the growing literature on how physical bodily expressions influence psychological processes, the authors propose that exerting physical strength decreases risk perceptions and increases preference for risky options by increasing perceptions of control or agency. The present research is based on the belief of "no pain, no gain", that when an individual exerts physical strength and effort, he believes he can be the agent in bringing about the desired outcome. Because of this automatic association between exerting physical strength and the sense of being in control of the outcome, the authors hypothesize that even in situations where the outcome is determined by chance and luck, individuals exerting effort feel they have more control and thus choose riskier, but more desirable, options. Furthermore, this research clarifies the distinction between physical exertion of strength, high- and low-power poses, and psychological power.
\end{abstract}

Keywords: physical strength, risk preference, sense of control, illusory control, risky choice

Risk is an inherent part of decision making. From the minute decisions of whether to carry an umbrella on a cloudy day, to the significant decisions of choosing which house to buy, varying degrees of risk are involved. Prior research has examined various factors that influence our perceptions of the risk in a given situation and our willingness to engage in risky behaviors. Situational factors (e.g. Kahneman and Tversky 1979; Novemsky and Dhar 2005), as well as the decision maker's internal states such as emotions (Lerner and Keltner 2001; Lerner et al. 2003) and cognitions (e.g. Anderson and Galinsky 2006; Freeman and Muraven 2010) have been examined. What remains unknown, however, is whether an individual's physiological state, and in particular, physical exertion of strength can influence risk perceptions

\footnotetext{
* Assistant Professor of Marketing, Smeal College of Business, Pennsylvania State University (eyk2@psu.edu)

** Assistant Professor of Marketing, School of Business, Hanyang University (hkahn@hanyang.ac.kr), Corresponding Author
} 
and engagement in risky behaviors. Building on the growing literature studying how physical bodily expressions influence psychological processes, we propose that exerting physical strength decreases risk perceptions and increases preference for risky options by increasing perceptions of control or agency.

More recently, the influence of physical actions, such as postures, on risk seeking has been examined. Studies show that the psychological feeling of power, which decreases risk perception and increases risky behaviors (Anderson and Galinsky 2006), is embodied, such that the adoption of open and expansive postures, referred to as high-power poses, can promote feelings of "being in charge" (Carney, Cuddy, and Yap 2010; Ranehill et al. 2015). While the connection between physical postures and the psychological perception of power has been shown, it remains ambiguous as to whether these high-power poses can actually influence risk taking behaviors. Carney, Cuddy, and Yap (2010) have shown that high-power poses cause individuals to feel powerful and to choose risky gambles compared to low-power poses (e.g. closed limb positions). Other research, however, shows that high-power poses do not affect preferences between safe and risky options (Ranehill et al. 2015). Moreover, the poses themselves do not require that physical strength be exerted. In fact, Ranehill et al. (2015) note that the potential discomfort from the prolonged posing time does not appear to influence the overall risk preference results.
It may even be possible that the low-power poses require more physical strength than the high-power poses depending on whether the individuals relaxed or flexed their muscles while holding the poses. Therefore, based on the previous research, we can conclude that (1) psychological power and the exertion of physical strength are not necessarily the same; and (2) that it is unclear whether physical acts can influence behavioral measures of risk seeking.

In the current research, we propose that physical exertion of strength increases risky behavior and that it does so because it leads to a sense of control over the outcome of a potentially risky situation. We build on the notion that using one's body and muscles to exert physical strength requires energy and is oftentimes effortful. The exertion of physical strength, whether it is hours of sweat and tears at the gym or at the office, is believed to bring about desirable outcomes in a myriad of domains. The ever popular proverb, "no pain, no gain," attests to this belief. It also engenders the belief that each individual can be the agent or an agentic force in maximizing the likelihood of bringing about the desired outcomes while keeping the undesired outcomes away. That is, my exertion of strength, my sweat, will help to ensure a desired positive outcome instead of an alternate undesired negative outcome. For instance, if I desire to have a fit physique, I can be the agent of that outcome by exerting physical strength and effort in the 
gym. If I desire to get good grades, my physical efforts of going to class and studying for exams (all of which require exertion of physical strength) play a significant role in the attainment of that desired outcome. As such, the numerous and varied instances where exerting physical strength is linked to my being agentic in bringing about a desired outcome, causes a link or association to form between the two. We know from prior research that associations or links between two entities can be formed through external cues in the environment (Morris, Menon, and Ames 2001) such as popular sayings, as well as through personal experiences and observations (Ross and Nisbett 1991). Furthermore, repeated coactivation between two entities can cause links between the two to become automatic (Bargh and Chartrand 1999; Goldsmith, Kim Cho, and Dhar 2012). Therefore, even momentary exertions of physical strength can automatically activate the belief that one has control over the outcome of a pending situation. As a result, we propose that even in situations where this belief is not applicable, the automatic association leads to the interesting phenomenon where individuals feel a sense of control, whether it be real or illusory (Langer 1975; Thompson, Armstrong, and Thomas 1998), over the outcome of a situation when they have exerted some form of physical strength. As a conservative test of this proposition, we examine situations where a desirable outcome is solely determined by chance and luck. We present individuals with a choice between an option that is desirable but risky and an option that is less desirable but less risky. Do I prefer the riskier, higher payoff gamble or the safer, lower payoff gamble? Do I risk forgoing buying insurance now or do I buy insurance now to protect against future losses? Although both situations feature outcomes which are primarily determined by chance and luck, rather than by personal exertion of strength, we expect that individuals exerting physical strength prior to making the decision, will feel more in control over the outcome, and choose the riskier option.

\section{Study $1 \mathrm{~A}$}

Studies $1 \mathrm{~A}$ and $1 \mathrm{~B}$ examine our main proposition that the physical exertion of strength will increase risk seeking. We manipulate the exertion of strength through various hand positions and then measure preferences for financial gambles that vary in risk.

\subsection{Method}

Participants were recruited to participate in one 15-minute laboratory session over the course of one day. Twenty-one undergraduate students participated in this study in exchange for partial course credit. Participants were informed that they would be completing a 
series of studies intended to measure motor skills and multi-tasking. The first task was designed to manipulate the exertion of physical strength. Drawing from prior literature that making a fist is mentally construed as an abbreviation of or preparation for the exertion of bodily force or physical strength (e.g. Gitin 1970; Schubert 2004), we operationalize the physical exertion of strength by the formation of a fist. The first task, adapted from Schubert (2004), was to simulate playing a game of rock-paper-scissors. They were shown pictures of each of the three moves. For example, for "rock", they saw a drawing of a closed fist, while for "paper" they saw a drawing of an open hand. Half of the participants were randomly assigned to the strength condition and were instructed to play "rock" and formed a fist with their non-writing hand. The other half of the participants was assigned to the neutral condition and were instructed to play "paper" and held their non-writing hand in an open position. Participants were instructed to hold their assigned hand position until told to release it. Then all participants were presented with two options that varied on likelihood of monetary winnings, but were equal in terms of expected value, and asked to indicate which option they would choose. Option A offered participants a sure gain of $\$ 5$, while option $B$ offered participants a $10 \%$ chance to win $\$ 50$ (and a $90 \%$ chance to win $\$ 0$ ). After choosing, participants released their assigned position.
Then all participants rated how tired they felt and how difficult it was to hold their hand in their assigned position $(1=$ not at all, $7=$ very). The main dependent variable was the percentage of participants choosing option B, the riskier option.

\subsection{Results}

We expected that participants who had been assigned to hold the "rock" position would be exerting more physical strength and thereby more likely to choose the riskier option B compared to those holding the "paper" position. Consistent with our proposition, participants in the strength condition were more likely to choose the riskier option compared to those in the neutral condition $\left(70 \%\right.$ vs. $18 \%, \chi^{2}(1)=$ 5.74, $p=.02$ ).

There were no differences in how tired participants felt $\left(\mathrm{M}_{\text {strength }}=4.50, \mathrm{SD}=1.65\right.$; $\mathrm{M}_{\text {neutral }}=5.00, \mathrm{SD}=2.79 ; \mathrm{t}(19)=.49, p>$ .63) or in how difficult they felt it was to hold their hand in their assigned position $\left(\mathrm{M}_{\text {strength }}=\right.$ $3.50, \mathrm{SD}=1.72 ; \mathrm{M}_{\text {neutral }}=3.00, \mathrm{SD}=1.90$; $t(19)=.63, p>.53)$. This helps to address the possibility that depletion increased preference for risk (Fischer, Kastenmuller, and Asal 2012). We also note that the strength condition, which was operationalized by the closed fist or the "rock", is more of a closed body gesture compared to the neutral condition, operationalized by the open hand or the "paper." This is in contrast 
to the typical understanding of high-power poses as being open and low-power poses as being closed (e.g. Carney, Cuddy, and Yap 2010), and serves to further distinguish physical strength from power.

\section{Study 1B}

\subsection{Method}

Participants were recruited to participate in one 15-minute laboratory session over the course of one day. Twenty-six undergraduate students participated in this study in exchange for partial course credit. Participants were informed that they would be completing a series of studies intended to measure motor skills and multitasking. The design of study 1B was identical to that of study $1 \mathrm{~A}$ except that participants randomly assigned to the neutral condition were told to play "scissors" with their non-writing hand (i.e. hold up two fingers) and to hold that position until instructed to release it. Then while participants were holding their respective positions ("rock" for strength condition and "scissors" for the neutral condition), they were presented with two gambles and asked to choose one. As in study $1 \mathrm{~A}$, the gambles varied on the amount of money and the likelihood of winning, but had equal expected value. Gamble A offered participants a $15 \%$ chance of winning
\$35 while Gamble B offered participants a $75 \%$ chance of winning $\$ 7$. After choosing, participants released their hand positions. All participants then rated how tired they felt and how difficult it was to hold their hand in their assigned position ( $1=$ not at all, $7=$ very $)$. The main dependent variable was the percentage of participants choosing Gamble A, the riskier gamble.

\subsection{Results}

As expected, participants in the strength condition were significantly more likely to choose the riskier gamble compared to those in the neutral condition $\left(50 \%\right.$ vs. $6 \%, \chi^{2}(1)=$ $6.64, p=.01)$. There were no differences in how tired participants felt $\left(\mathrm{M}_{\text {strength }}=4.50, \mathrm{SD}\right.$ $=1.65 ; \mathrm{M}_{\text {neutral }}=5.50, \mathrm{SD}=2.63 ; \mathrm{t}(24)=$ $1.07, p>.29$ ) or in how difficult they felt it was to hold their hand in their assigned position $\left(\mathrm{M}_{\text {strength }}=3.50, \mathrm{SD}=1.72 ; \mathrm{M}_{\text {neutral }}=4.44\right.$, $\mathrm{SD}=2.53 ; \mathrm{t}(24)=1.03, p>.31)$.

Taken together, Studies $1 \mathrm{~A}$ and $1 \mathrm{~B}$ show that individuals exerting physical strength consistently prefer to choose the riskier options that offer a lower likelihood of winning larger financial gains over ones that offer a higher likelihood of winning smaller financial gains. These findings provide initial support for our proposition that exerting physical strength, by making a fist, increases preference for risk. In the next studies, we begin to explore why we 
observe this phenomenon.

\section{Study 2}

Study 2 examines how physical strength affects perceptions of the likelihood of attaining a desired outcome. We expect to find that individuals exerting physical strength will perceive a greater likelihood of attaining a desired outcome.

\subsection{Method}

Participants were recruited to participate in one 15-minute laboratory session held over the course of two days. Forty-four undergraduate and graduate students participated in this experiment and were compensated with a small amount of money. As in Study 1B, participants were randomly assigned to strength and neutral conditions under the guise of playing the "rock-paper-scissors" game. Participants assigned to the strength condition formed a fist to play "rock" while those assigned to the neutral condition held up two fingers to play "scissors". While still holding their respective hand positions, all participants were presented with a scenario where they had a $10 \%$ chance of winning a \$1000 lottery drawing. They rated their perceived likelihood of winning the $\$ 1000$ on a 7 point scale $(1=$ very low, $7=$ very high). All participants then rated how tired they felt and how difficult it was to hold their hand in their assigned position $(1=$ not at all, 7 = very).

\subsection{Results}

As expected, exerting physical strength decreased the perception of risk in the financial scenario. Individuals in the strength condition thought that they were more likely to win the money compared to those in the neutral condition $\left(\mathrm{M}_{\text {strength }}=5.39, \mathrm{SD}=1.78 ; \mathrm{M}_{\text {neutral }}=4.29\right.$, $\mathrm{SD}=1.65 ; \mathrm{t}(42)=-2.13, p=.04)$. Even though the chances of winning the money were fixed at 10\% for all individuals, those exerting physical strength by forming a fist felt as though they were more likely to win. There were no differences in how tired participants felt $\left(\mathrm{M}_{\text {strength }}=5.78, \mathrm{SD}=1.98 ; \mathrm{M}_{\text {neutral }}=\right.$ $5.67, \mathrm{SD}=1.88 ; \mathrm{t}(42)=-.20, p>.44)$ or in how difficult they felt it was to hold their hand in their assigned position $\left(\mathrm{M}_{\text {strength }}=3.91, \mathrm{SD}\right.$ $=2.26 ; \mathrm{M}_{\text {neutral }}=3.62, \mathrm{SD}=2.16 ; \mathrm{t}(42)=$ $-.44, p>.96)$.

\subsection{Discussion}

Study 2 builds on the findings from Studies $1 \mathrm{~A}$ and $1 \mathrm{~B}$ to show that even subtle exertions of physical strength can change the way in which individuals perceive outcomes that are determined largely by chance. Next, we examine 
more directly the proposed psychological process: the exertion of physical strength increases individuals' perception of the likelihood of favorable outcomes (Study 2) and increases preference for riskier gambles (Studies $1 \mathrm{~A}$ and 1B) because of the increased feeling of agency in the attainment (avoidance) of the desired (undesired) outcome.

Studies $3 \mathrm{~A}$ and $3 \mathrm{~B}$ examine this process through an experimental causal chain design. This design is particularly recommended for cases such as ours, where measuring as well as manipulating the process is simple and straightforward, because it leverages the power of experiments to infer causality (Spencer, Zanna, and Fong 2005). Our proposed chain is that exertion of physical strength triggers the feeling of agency, and that this feeling of agency in turn influences the perception of risk or the likelihood that a particular outcome will occur. Following the procedures set by Spencer, Zanna, and Fong (2005), we first manipulate the exertion of physical strength and then observe the effect on perceived agency over outcome in Study 3A. In Study 3B, we manipulate perceived agency over outcome and observe the effect on perception of risk.

\section{Study 3A}

The purpose of Study 3A is three-fold. First, it revalidates the findings from Studies 1 and 2 that the exertion of physical strength leads to the preference for risky behavior using a different manipulation for physical strength. Although we think that making fist can activate or simulate the intent of using bodily force or strength (Schubert 2004), one may argue that it is not safe to assume that holding a fist simulates actually exerting physical force. Study 3A directly tests the effect of physical strength by manipulating actual strength exertion. Second, contrary to the previous studies that used desirable outcomes (e.g. winning money) that individuals want to attain, Study 3A uses negative outcomes that individuals will want to avoid. Using negative outcomes allows us to address a potential alternative explanation that exerting physical strength induces an actionoriented mindset that causes individuals to take action regardless of the risk associated with it. Given that our manipulation of physical strength thus far was based on the notion that making a fist signals the intent of using bodily force (Schubert 2004), it is possible that that particular gesture primes a general action goal or orientation (Huang et al. 2011), which has been associated with impulsivity, and reduced inhibitory control processes (Albarracín et al. 2008; Hepler and Albarracín 2013). By using negative outcomes, we can disentangle whether exerting physical strength simply primes individuals to take action, whatever that might be, or whether exerting strength leads to risky behaviors, even 
when the risky behavior takes the form of inaction. An action-orientation story would predict that an individual primed with physical strength would choose to take action and thereby engage in behaviors that would minimize the potential for the negative outcome. Contrary to this action-orientation prediction and consistent with our proposition, we expect that individuals exerting physical strength will demonstrate preference for risk by not engaging in behaviors that will minimize the negative outcome. Third, we demonstrate the first part of the causal chain that physical exertion leads to enhanced feelings of agency over the pending outcome.

\subsection{Method}

Participants were recruited to participate in one 30-minute session over the course of two days. One hundred and two undergraduate students participated in this study in exchange for partial course credit. Participants were randomly assigned to the strength or neutral conditions. Participants in the strength condition firmly grasped a pencil in their clenched fists, while those in the neutral condition lightly held a pencil between their thumb and index fingers (adapted from Hung and Labroo 2011). While holding the pencil as directed, they imagined purchasing auto insurance and indicated whether they wanted to include injury coverage at an additional cost. Again, the notion is that not taking action by not purchasing the additional coverage would demonstrate a preference for risk. Afterwards, they rated the extent to which they personally had control over what happens in the future while driving their car ( $1=$ very little, $7=$ a lot $)$. Lastly, participants were asked to release the pencil and indicated how much physical strength they had exerted while holding the pencil, how tired they felt, and how difficult it was to hold the pencil $(1=$ none/not at all, $7=$ very much).

\subsection{Results}

The manipulation check confirmed that the individuals grasping the pencil firmly in their clenched fist exerted more physical strength than those holding the pencil lightly between their two fingers $\left(M_{\text {strength }}=4.59, \mathrm{SD}=2.38\right.$; $\mathrm{M}_{\text {neutral }}=3.22, \mathrm{SD}=2.09 ; \mathrm{t}(100)=3.10, p=$ .003). Furthermore, there were no differences in how tired participants felt $\left(\mathrm{M}_{\text {strength }}=4.67\right.$, $\mathrm{SD}=2.17 ; \mathrm{M}_{\text {neutral }}=4.47, \mathrm{SD}=2.36 ; \mathrm{t}(100)$ $=.44, p>.66$ ) or in how difficult they felt it was to hold the pencil as instructed $\left(\mathrm{M}_{\text {strength }}=\right.$ 2.92, $\mathrm{SD}=2.10 ; \mathrm{M}_{\text {neutral }}=2.73, \mathrm{SD}=2.24$; $\mathrm{t}(100)=.46, p>.64)$.

Consistent with our proposition, participants in the strength condition were less likely to select the additional injury insurance compared to those in the neutral condition (61\% vs. $80 \%$, $\left.\chi^{2}(1)=4.72, p=.03\right)$. Thus we rule out the action-orientation mindset story and demonstrate that exerting physical strength increases 
preference for risky behaviors regardless of whether individuals are taking active measures to approach risk or abstaining from precautionary measures to avoid risk. Furthermore as expected, those in the strength condition reported a greater sense of being in control of potential outcomes while driving their car than those in the neutral condition $\left(\mathrm{M}_{\text {strength }}=6.06, \mathrm{SD}=\right.$ $1.72 ; \mathrm{M}_{\text {neutral }}=5.20, \mathrm{SD}=1.87 ; \mathrm{t}(100)=$ 2.42, $p=.02$ ). This result demonstrates that physical strength leads to increased feelings of agency and control, which supports the first part of our causal chain. Study 3B tests the second part of our causal chain that feelings of agency and control lead to preference for risk.

\section{Study 3B}

\subsection{Method}

Participants were recruited to participate in one 30-minute session over the course of two days. One hundred and eight undergraduate students participated in this study in exchange for extra course credit. Participants were randomly assigned to either a high agency condition or a neutral condition. Based on prior research that personal choice provides feelings of control and agency (e.g. Averill 1973; Inesi et al. 2011; Lefcourt 1973), those in the high control condition were presented with a grocery shopping task where they could choose which items they would like to purchase. The shopping task guided participants through a hypothetical grocery store and allowed them to choose as many items as they would like from each of three sections. For example, in the produce section, participants saw a list of available items such as tomatoes, zucchini, onions, bananas, and clicked and dragged desired items into their virtual shopping cart. This choice task was repeated for the other two sections. Participants in the neutral condition were presented with a similar grocery shopping task, but instead of choosing items from each section, they rank ordered the items in terms of popularity among fellow college students. For example, in the produce section, they would rank the items from most popular to least popular. This ranking task was repeated for the other two sections. Next, all participants were presented with a reading passage about the potential risks and dangers of gambling. After reading the passage, participants were asked provide an appropriate title for the passage and to rate how risky gambling is ( $1=$ not at all, 9 = very). Lastly as a manipulation check, participants were asked to think back to the grocery task and indicate how much control they felt they had ( $1=$ not at all, $9=$ very much). 


\subsection{Results}

The manipulation check confirmed that those making choices in the high control condition felt a greater sense of control than those rank ordering items in the neutral condition $\left(\mathrm{M}_{\text {high control }}\right.$ $=7.48, \mathrm{SD}=1.63 ; \mathrm{M}_{\text {neutral }}=6.37, \mathrm{SD}=$ $2.26 ; t(106)=2.96, p=.004)$.

We predicted that those in the high control condition would perceive lower levels of risk associated with gambling compared to those in the neutral condition. Consistent with our expectations, the high control participants felt that gambling was less risky than the neutral condition participants $\left(\mathrm{M}_{\text {high control }}=6.77, \mathrm{SD}=\right.$ $1.51 ; \mathrm{M}_{\text {neutral }}=7.37, \mathrm{SD}=1.71 ; \mathrm{t}(106)=$ 1.94, $p=.055)$.

These results support the final causal link in our model that feelings of agency and control lead to a reduced perception of risk. Studies $3 \mathrm{~A}$ and $3 \mathrm{~B}$ together, demonstrate evidence of our proposition that the exertion of physical strength increases feelings of agency and control over the outcome, which in turn reduces the perceived riskiness of potentially dangerous behaviors and outcomes.

\section{General Discussion}

We demonstrate that physical acts, and in particular, the exertion of physical strength can decrease perceptions of risk and increase preference for risky behavior. A causal chain of experiments confirms that the physical exertion of strength leads to a (illusory) sense of agency and control over a pending outcome, which in turn increases preference for risk. The current research contributes to the literature on risk-taking and expands our understanding of the link between physical bodily actions and psychological processes in influencing risk perceptions and preference for risky behaviors.

We note that our current research, while related to the prior work on power and risk, is quite different. In the earlier work, the notion of power has largely been studied as a social construct where an individual or group of individuals has or perceives to have power over other individuals usually by having more access to and more control over desirable resources (Magee and Galinsky 2008). This understanding of power has led to research showing that when individuals recall instances of having power or imagine being the boss of a group of individuals, it leads to optimistic risk perceptions, which in turn promote risky behaviors (e.g., Anderson and Galinsky 2006). It is plain to see that actually having or perceiving to have social power over other individuals is a psychological experience and does not necessarily require the exertion of physical strength. Rather, the main requirement for social power is that an individual feels as though he has control over other individuals independent of 
his actual structural or hierarchical position (Galinsky, Rucker, and Magee 2015). Consistent with this notion, Kim and McGill (2011) find that people primed with high (social) power, perceive less risk when abstract dangers such as gambling and cancer are anthropomorphized. Because social power is about control over other human individuals, when these inanimate nonhuman dangers take on characteristics of humans, the individual feels that they have control over the dangers and are perceived as less risky. Thus, even though high power can be associated with feelings of agency (Fast et al. 2009; Rucker, Galinsky, and Dubois 2012) and in a sense overlap with the physical exertion of strength, it has primarily been understood and examined within a social context. Future research can examine whether exerting strength, even momentarily, can produce not only the feeling of agency in bringing about a desired outcome, but also the feeling of mastery and control in a social context.

Lastly, consider the athlete who celebrates the successful execution of an odds-defying trick with an emphatic fist pump. Perhaps even after the fact, the quick exertion of strength can retroactively reinforce the belief of her agency in having just brought about the desired (but luck-driven, even for the highly skilled individual) outcome, and increase belief for similar outcomes in the near future.

〈Received January 29. 2020〉

〈Accepted March 24. 2020〉

\section{References}

Albarracín, Dolores, Ian M. Handley, Kenji Noguchi, Kathleen C. McCulloch, Hong Li, Joshua Leeper, Rick D. Brown, and William P. Hart (2008), "Increasing and Decreasing Motor and Cognitive Output: A Model of General Action and Inaction Goals," Journal of Personality and Social Psychology, 95(3), 510-523.

Anderson, Cameron, and Adam D. Galinsky (2006), "Power, Optimism, and Risk-taking," European Journal of Social Psychology, 36 (4), 511-536.

Averill, James R. (1973), "Personal Control over Aversive Stimuli and Its Relationship to Stress," Psychological Bulletin, 80(4), 286-303.

Bargh, John A., and Tanya L. Chartrand (1999), "The Unbearable Automaticity of Being," American Psychologist, 54(7), 462-479.

Carney, Dana R., Amy JC Cuddy, and Andy J. Yap (2010), Power Posing: Brief Nonverbal Displays Affect Neuroendocrine Levels and Risk Tolerance," Psychological Science, 21 (10), 1363-1368.

Fast, Nathanael J., Deborah H. Gruenfeld, Niro Sivanathan, and Adam D. Galinsky (2009), "Illusory Control: A Generative Force behind Power's Far-reaching Effects," Psychological Science, 20(4), 502-508. Fischer, Peter, Andreas Kastenmüller, and 
Kathrin Asal (2012), “Ego Depletion Increases Risk-taking," The Journal of Social Psychology, 152(5), 623-638.

Freeman, Nicholas, and Mark Muraven (2010),

"Self-control Depletion Leads to Increased Risk Taking," Social Psychological and Personality Science, 1 (2), 175-181.

Galinsky, Adam D., Derek D. Rucker, and Joe C. Magee (2015), "Power: Past Findings, Present Considerations, and Future Directions," in APA Handbook of Personality and Social Psychology, Vol. 3, Mario Mikulincer and Phillip R. Shaver, eds., Washington, D.C.: American Psychological Association, 421-460.

Gitin, Sharon R. (1970), “A Dimensional Analysis of Manual Expression," Journal of Personality and Social Psychology, 15 (3), 271-277.

Goldsmith, Kelly, Eunice Kim Cho, and Ravi Dhar (2012), "When Guilt Begets Pleasure: The Positive Effect of a Negative Emotion," Journal of Marketing Research, 49(6), 872-881.

Hepler, Justin, and Dolores Albarracín (2013), "Complete Unconscious Control: Using (In) Action Primes to Demonstrate Completely Unconscious Activation of Inhibitory Control Mechanisms," Cognition, 128(3), 271-279. Huang, Li, Adam D. Galinsky, Deborah H. Gruenfeld, and Lucia E. Guillory (2011), "Powerful Postures versus Powerful Roles: Which is the Proximate Correlate of
Thought and Behavior?," Psychological Science, 22(1), 95-102.

Hung, Iris W., and Aparna A. Labroo (2011), "From Firm Muscles to Firm Willpower: Understanding the Role of Embodied Cognition in Self-Regulation," Journal of Consumer Research, 37(6), 1046-1064.

Inesi, M. Ena, Simona Botti, David Dubois, Derek D. Rucker, and Adam D. Galinsky (2011), "Power and Choice: their Dynamic Interplay in Quenching the Thirst for Personal Control," Psychological Science, 22(8), 1042-1048.

Kahneman, Daniel, and Amos Tversky (1979), "Prospect Theory: An Analysis of Decision under Risk," Econometrica, 47(2), 263-292. Langer, Ellen J. (1975), "The Illusion of Control," Journal of Personality and Social Psychology, 32(2), 311-328.

Lefcourt, Herbert M. (1973), "The Function of the Illusions of Control and Freedom," American Psychologist, 28(5), 417-425.

Lerner, Jennifer S., and Dacher Keltner (2001), "Fear, Anger, and Risk," Journal of Personality and Social Psychology, 81(1), 146-159.

Lerner Jennifer S., Roxana M. Gonzalez, Deborah A. Small, and Baruch Fischhoff (2003), "Effects of Fear and Anger on Perceived Risks of Terrorism: A National Field Experiment," Psychological Science, 14(2), 144-150.

Kim, Sara, and Ann L. McGill (2011), "Gaming 
with Mr. Slot or Gaming the Slot Machine? Power, Anthropomorphism, and Risk Perception," Journal of Consumer Research, 38(1), 94-107.

Magee, Joe C., and Adam D. Galinsky (2008), "Social Hierarchy: The Self-Reinforcing Nature of Power and Status," Academy of Management Annals, 2(1), 351-398.

Morris, Michael W., Tanya Menon, and Daniel R. Ames (2001), "Culturally Conferred Conception of Agency: A Key to Social Perception of Persona, Groups, and Other Actors," Journal of Personality and Social Psychology, 5(2), 169-182.

Novemsky, Nathan, and Ravi Dhar (2005), "Goal Fulfillment and Goal Targets in Sequential Choice," Journal of Consumer Research, 32(3), 396-404.

Ranehill Eva, Anna Dreber, Magnus Johannesson, Susanne Leiberg, Sunhae Sul, and Roberto A. Weber (2015), "Assessing the Robustness of Power Posing: No Effect on Hormones and Risk Tolerance in a Large Sample of Men and Women," Psychological Science, 26(5), 653-656.
Ross, Lee, and Richard E. Nisbett (1991), The Person and the Situation: Perspectives of Social Psychology. New York, NY: McGraw Hill.

Rucker, Derek D., Adam D. Galinsky, and David Dubois (2012), "Power and Consumer Behavior: How Power Shapes Who and What Consumers Value," Journal of Consumer Psychology, 22(3), 352-368.

Schubert, Thomas W. (2004), "The Power in Your Hand: Gender Differences in Bodily Feedback from Making a Fist," Personality and Social Psychology Bulletin, 30(6), 757-769.

Spencer, Steven J., Mark P. Zanna, and Geoffrey T. Fong (2005), "Establishing a Causal Chain: Why Experiments are Often More Effective than Mediational Analyses in Examining Psychological Processes," Journal of Personality and Social Psychology, 89 (6), 845-851.

Thompson, Suzanne C., Wade Armstrong, and Craig Thomas (1998), "Illusions of Control, Underestimations, and Accuracy: A Control Heuristic Explanation," Psychological Bulletin, 123(2), 143-161. 